\title{
Exame imuno-histoquímico na biópsia de medula óssea: uma importante ferramenta complementar à morfologia
}

Primeira submissão em 25/06/11 Última submissão em 09/09/11 Aceito para publicação em 19/09/11 Publicado em 20/12/11

\section{Immunohistochemistry in bone marrow biopsy: an important complementary tool for morphology}

Maria Cláudia Nogueira Zerbini

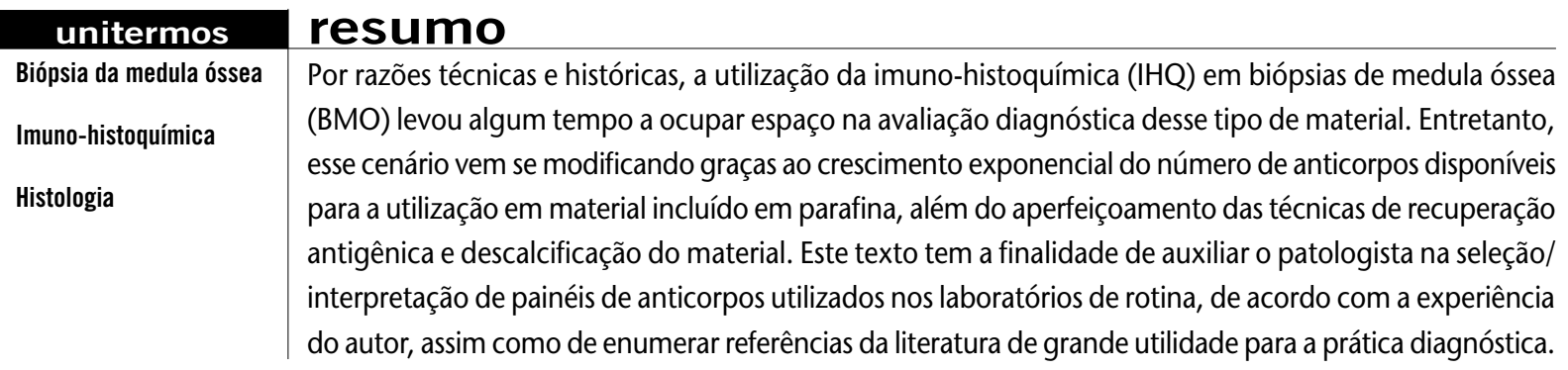

\section{abstract}

Due to historical and technical reasons, the use of immunohistochemistry (IHC) in bone marrow biopsies $(B M B)$ has not till recently been introduced in diagnostic evaluation. However, this scenario has changed owing to the exponential growth in the number of antibodies available for paraffin-embedded material and the development of techniques for antigen retrieval and material decalcification. Not only does this text aim to assist pathologists in the selection/interpretation of antibody panels used in routine laboratories, but it also lists literature references highly useful for diagnostic practice in accordance with the author's experience. key words

Bone marrow biopsy

Immunohistochemistry

Histology 


\section{Introdução}

Por razões técnicas e históricas, a utilização da imuno-histoquímica (IHQ) em biópsias de medula óssea (BMO) levou algum tempo a ocupar espaço na avaliação diagnóstica desse tipo de material. Entretanto, esse cenário vem se modificando graças ao crescimento exponencial do número de anticorpos disponíveis para a utilização em material incluído em parafina, além do aperfeiçoamento das técnicas de recuperação antigênica e descalcificação do material. Este texto tem a finalidade de auxiliar o patologista na seleção/interpretação de painéis de anticorpos utilizados nos laboratórios de rotina, de acordo com a experiência do autor, assim como de enumerar referências da literatura de grande utilidade para a prática diagnóstica ${ }^{(1-12)}$.

\section{Aspectos técnicos}

Os processos de fixação e descalcificação são fundamentais para o sucesso dos procedimentos de IHQ. Embora alguns fixadores mercuriais, como Zenker e B5, tenham sido muito utilizados com o intuito de se obter excelência nos detalhes morfológicos, eles não têm sido considerados adequados para a análise molecular, além de prejudicar a IHQ quando o tempo de fixação excede o tempo preconizado de 2 horas e meia. Dessa forma, tem-se difundido cada vez mais o uso do formol tamponado, com excelentes resultados não só para análise morfológica e IHQ, mas também para as técnicas moleculares envolvendo DNA e RNA. Da mesma maneira, várias técnicas de descalcificação têm sido utilizadas, sendo os fixadores de base ácida e os agentes quelantes de cálcio, como o ácido etilenodiaminotetracético (EDTA), os mais utilizados, respectivamente, nos EUA e na Europa. Nossa experiência tem demonstrado bons resultados com a descalcificação pelo EDTA em material fixado em formol tamponado. Embora a imunorreatividade seja, em geral, boa nas BMO, as reações devem obedecer a protocolos próprios otimizados para essa finalidade. Um programa de controle de qualidade especificamente direcionado para IHQ nas BMO é altamente desejável.

O número e a escolha dos anticorpos necessários dependem do tipo de doença e do questionamento clínico. Por exemplo, a classificação de uma neoplasia identificada primariamente na medula óssea (MO) requer um painel de anticorpos mais amplo, enquanto a confirmação da infiltração por um linfoma já diagnosticado previamente, a avaliação da resposta terapêutica, a detecção de recidiva e a doença residual mínima, geralmente, são possíveis com um número restrito de anticorpos. Deve-se lembrar ainda que alguns marcadores usados de rotina no painel de linfomas, como CD15, CD43 e CD68 (KP1), mostram ampla reatividade com células mieloides, sendo, portanto, menos adequados na $\mathrm{MO}$ em relação aos linfonodos. Na Tabela 1 estão relacionados os anticorpos úteis comumente utilizados para a IHQ da MO.

As indicações mais comuns de IHQ em BMO incluem:

- caracterização dos processos linfoproliferativos crônicos/doenças proliferativas imunossecretoras;

- caracterização das doenças proliferativas mieloides: $\sqrt{ }$ síndrome mielodisplásica (SMD)/leucemias agudas (LAs);

$\sqrt{ }$ neoplasia mieloproliferativa crônica (NMPC);

$\sqrt{ }$ entidades integrantes do grupo das NMPCs/ SMD;

- investigação de doença metastática;

- alterações reativas da $\mathrm{MO}$ - identificação de agentes infecciosos.

\section{Caracterização dos processos linfoproliferativos crônicos/doenças proliferativas imunossecretoras} Regras gerais

Complementar sempre o exame histológico com o exame IHQ, exceto no caso do linfoma folicular (LF) e do linfoma de Hodgkin clássico, nos quais a ausência de indicadores morfológicos exclui a necessidade de exame IHQ complementar. Nesses casos, recomendam-se secções seriadas do bloco de parafina para eventual detecção de pequenos focos de comprometimento não visíveis ao exame da primeira lâmina.

É essencial e necessária a informação ao patologista do tipo de linfoma diagnosticado anteriormente, no caso de avaliação de estadiamento, recidiva ou suspeita de evolução da doença para uma forma mais agressiva.

É necessária a informação sobre medicamentos em uso, como fatores de crescimento (eritropoietina, fator estimulador de colônias de granulócitos [GCSF] e outros), quimioterápicos ou biológicos, como anti-CD20, pois podem ser responsáveis por importantes alterações na $\mathrm{MO}$ que podem ser confundidas com neoplasia. No caso do anti-CD20, em $5 \%$ das biópsias está presente expressivo infiltrado linfoide T (CD3/CD4/FOXP3 positivos). Nessa situação, recomenda-se utilizar PAX5 ou CD79a como alternativa para identificação das células $B$. 


\section{Tabela 1 Sugestão de anticorpos primários de utilidade na avaliação de BMO}

\begin{tabular}{|c|c|c|}
\hline Antígeno & Clone (ref.1/HC) & Expressão celular relevante \\
\hline CDla & MTB-1 & Célula T tímica, CL \\
\hline CD2 & AB75 & Célula T tímica, célula NK \\
\hline CD3 & F7.2.38 & Célula T madura \\
\hline CD4 & IF6 & Célula T auxiliadora \\
\hline CD5 & $4 \mathrm{C7}$ & Célula T madura \\
\hline CD7 & 272 & Célula T tímica \\
\hline CD8 & C8/144B & Célula T citotóxica \\
\hline CD10 & $56 C 6$ & Célula B CG, CPL \\
\hline CD15 & MMA & Granulócitos, monócitos, $\mathrm{CH}$ \\
\hline CD20 & L26 & Célula B madura, exceto o plasmócito \\
\hline CD21 & IF8 & Subgrupo de células B, CFD \\
\hline CD23 & $|B| 2$ & Subgrupo de células $B, C F D, C L$ \\
\hline CD30 & BerH2 & Células $\mathrm{B} / \mathrm{T}$ ativadas, $\mathrm{CH}$ \\
\hline CD34 & QBEnd-10 & Célula progenitora, célula endotelial \\
\hline CD43 & MT1 & Célula T e mieloides \\
\hline CD45 & X16/99 & Pan-leucocitário \\
\hline CD56 & IB6 & Células NK/T/MM \\
\hline CD61 & Y2/51 & MGC, célula endotelial \\
\hline CD68R & PGM-1 & Monócitos/macrófagos, CL \\
\hline CD68 & KP1 & Monócitos/macrófagos, células mieloides, CL \\
\hline CD79a & HM57 & Célula $B$, plasmócitos \\
\hline CD117 & c-kiit & Célula progenitora, promielócitos, mastócitos \\
\hline CD138 & MI15(4) & Plasmócito \\
\hline ALK1 & $\operatorname{ALK1(2)}$ & LGCA \\
\hline BCL-2 & 124(3) & Subgrupo de linfócitos \\
\hline BCL-6 & P1F6 & CG, célula $B$ ativada \\
\hline Ciclina D1 & SP4 & Ciclo celular (MM, LCM) \\
\hline DBA.44 & DBA.44 & Hairy cells, subgrupos de células B \\
\hline Granzima B & GrB-7 & Célula T citotóxica \\
\hline Glicoforina A & JC159 & Linhagem eritrocítica \\
\hline Lisozima & Policlonal & Monócitos \\
\hline Triptase dos mastócitos & AA1 & Mastócitos \\
\hline MUM-1 & MUM-1p & Plasmócitos, células B pós-CG \\
\hline Mieloperoxidase & MPO-7 & Linhagem granulocítica \\
\hline PAX-5 & Policlonal & Linfócitos B maduros e CPL-B, CH (fraco) \\
\hline P27 & F8 & Ciclo celular \\
\hline TdT & Policlonal & Células $B / T$ imaturas \\
\hline TIA-1 & 2G9 & Célula T citotóxica \\
\hline VS38c & VS38c & Plasmócito \\
\hline Kappa & R10-21-F3 & Cadeia leve da Ig \\
\hline Lambda & $\mathrm{N} 10 / 2$ & Cadeia leve da Ig \\
\hline $\lg G$ & 7701 & IgG \\
\hline $\lg A$ & NIF2 & $\lg A$ \\
\hline IgM & $\mathrm{R} 1 / 69$ & $\lg M$ \\
\hline $\lg D$ & Policlonal & $\lg D$ \\
\hline
\end{tabular}

BMO: biópsias de medula óssea; Ig: imunoglobulina; CL: célula de Langerhans; NK: natural killer; CG: centro germinativo; CPL: célula precursora linfoide; CH: célula de Hodgkin; CFD: célula folicular dendrítica; MM: mieloma múltiplo; MGC: megacariócitos; LGCA: linfoma de grandes células anaplásicas; LCM: linfoma de células do manto. 
Nos nódulos linfoides reacionais (idosos e jovens com distúrbios autoimunes, pacientes portadores da síndrome da imunodeficiência adquirida [AIDS]), a proporção de linfócitos T/B é de cerca de 5/1 com CD8 >> CD4.

Hematogonias são células precursoras B (CD20/PAX5/ CD79a/CD10/TdT positivos) em estágios variáveis de maturação, que podem aparecer em grande número (até $50 \%$ das células nucleadas) na $\mathrm{MO}$ em fase de recuperação imune e hematopoética após quimioterapia ou transplante de $\mathrm{MO}$, em doenças congênitas ou adquiridas, comprometendo qualquer uma das três séries hematopoéticas, ou em portadores de neoplasias. São mais comuns em crianças, mas podem ocorrer também nos adultos. É importante seu reconhecimento pela possibilidade do equívoco diagnóstico com a leucemia linfoide aguda (LLA); a observação fornecida pela citometria de fluxo (CMF) e os informes clínicos podem contribuir para a interpretação correta nessa situação.

\section{Linfomas/leucemias de linfócitos pequenos}

Nesse caso, devem-se utilizar o painel e a interpretação descritos na Tabela 2.

\section{- Pitfalls}

O LF frequentemente não expressa CD10, BCL-6 e CD21 (células foliculares dendríticas) quando infiltra a MO. O linfoma difuso de grandes células (LDGCB) pode infiltrar a MO com padrão discordante de células pequenas peritrabeculares, não sendo acompanhado de prognóstico desfavorável, como ocorre nos casos de infiltração com fenótipo concordante de células grandes.

Outra situação comum entre os processos linfoproliferativos B, em que a IHQ na BMO pode ter papel importante, refere-se ao diagnóstico diferencial entre a tricoleucemia $(\mathrm{HCL})$ e o linfoma da zona marginal esplênico (LZME). A Tabela 3 apresenta uma seleção de marcadores úteis para essa situação, utilizando-se a IHQ e a CMF.

\section{Linfomas/leucemias de células T/natural killer (NK)}

As Tabelas 4 e 5 apresentam, respectivamente, os anticorpos úteis na investigação dos processos linfoproliferativos de células $\mathrm{T} / \mathrm{NK}$ e a frequência de comprometimento da $\mathrm{MO}$ por seus diferentes subtipos.

Nas doenças que se apresentam com quadro leucêmico como manifestação primária, a imunofenotipagem por CMF pode ser um recurso importante para o diagnóstico.

O exame IHQ é fundamental para o diagnóstico do comprometimento da $\mathrm{MO}$ pelos linfomas $\mathrm{T}$ que não se apresentam primariamente com manifestação leucêmica, sendo, muitas vezes, representados por alterações sutis, de difícil identificação por análise morfológica isolada. Os linfomas de grandes células anaplásicas (LGCAs) ALK+ e ALK- são exemplos importantes dessa situação, apresentando, muitas

\begin{tabular}{|c|c|c|c|c|c|c|c|}
\hline Diagnóstico & $\begin{array}{l}\text { Padrão de infiltração } \\
\text { + comum/- comum }\end{array}$ & CD20 & CD5 & CD23 & $\begin{array}{c}\text { Ciclina } \\
\text { D1 }\end{array}$ & $\begin{array}{l}\text { Marcadores } \\
\text { adicionais }\end{array}$ & Comentários \\
\hline LLC/LL & $\mathrm{NI} / \mathrm{I} / \mathrm{D}$ & $\begin{array}{c}+ \\
\text { fraco }\end{array}$ & + & + & - & CD43+/ZAP70+/- & $\begin{array}{l}\text { Centros proliferativos } \\
\text { ocasionais }\end{array}$ \\
\hline LCM & NI/NP/I & + & + & - & + & CD43+/p27- & \\
\hline LZM & $\mathrm{NI}$ & + & - & - & - & CD43-/+ & CGs ocasionais \\
\hline LLPL & $\mathrm{NI} / \mathrm{NP}$ & + & $-/+$ & - & - & Ig citoplasmática & $\uparrow$ mastócitos \\
\hline LZME & $\mathrm{NI} / \mathrm{S}$ & + & - & - & - & lg+ & \\
\hline LF & NP & + & - & - & - & $\begin{array}{l}\text { CD10+/BCL-6+/ } \\
\text { CD43+ }\end{array}$ & $\begin{array}{l}\text { Folículos verdadeiros } \\
\text { raros }\end{array}$ \\
\hline $\mathrm{HCL}$ & I/D & + & - & - & $+/-$ & $\begin{array}{l}\text { DBA44+/CD25+, } \\
\text { TRAP+, anexina A1+ }\end{array}$ & $\begin{array}{l}\text { Fibrose, infiltrado } \\
\text { frouxo }\end{array}$ \\
\hline MM & Variável & $-1+$ & -1 & - & $-/+$ & $\begin{array}{l}\text { Cadeias leves Ig } \\
\text { CD138+, CD56+/, } \\
\text { VS38c+, CD38+ }\end{array}$ & \\
\hline
\end{tabular}

IHQ: imuno-histoquímico; LLC/LL: leucemia linfoide crônico/linfoma linfocítico; LCM: linfoma de células do manto; LZM: linfoma da zona marginal; LLPL: linfoma linfoplasmocítico; LZME: linfoma da zona marginal esplênico; LF: linfoma folicular; HCL: tricoleucemia; MM: mieloma múltiplo; NI: nodularintertrabecular; CGs: centros germinativos; I: intersticial; D: difuso; NP: nodular-peritrabecular; S: sinusoidal. 


\section{Marcadores imunofenotípicos (CMF e IHQ) úteis para o diagnóstico diferencial de HCL, LZME e}

Tabela 3 outros linfomas de linfócitos pequenos

\begin{tabular}{|c|c|c|c|c|c|c|c|}
\hline & LZME & LLC/LL & LCM & LF & $\mathrm{HCL}$ & HCL-V & L.MALT \\
\hline \multicolumn{8}{|l|}{ CMF } \\
\hline Ig superfície forte & +++ & $+/-$ & +++ & +++ & +++ & +++ & +++ \\
\hline CD5 & + & +++ & +++ & Neg. & Neg. & Neg. & Neg. \\
\hline CD23 & + & t++ & Neg. & + & Neg. & Neg. & Neg. \\
\hline FMC7 & +++ & Neg. & +++ & +++ & +++ & +++ & +++ \\
\hline CD11c & ++ & Neg. & Neg. & Neg. & +++ & +++ & Neg. \\
\hline CD103 & Neg. & Neg. & Neg. & Neg. & +++ & ++ & Neg. \\
\hline CD123 & Neg. & Neg. & Neg. & Neg. & +++ & Neg. & Neg. \\
\hline CD25 & + & Neg. & Neg. & Neg. & +++ & Neg. & Neg. \\
\hline CD27 & ++ & +++ & +++ & +++ & Neg. & ++ & + \\
\hline \multicolumn{8}{|l|}{$\mathrm{IHQ}$} \\
\hline DBA44 & ++ & + & Neg. & Neg. & +++ & +++ & Neg. \\
\hline $\operatorname{lgM} / \operatorname{lgD}$ & +++ & +++ & Neg. & + & +++ & + & + \\
\hline CD10 & Neg. & Neg. & Neg. & +++ & Neg. & Neg. & Neg. \\
\hline BCL-6 & Neg. & Neg. & Neg. & +++ & Neg. & Neg. & Neg. \\
\hline CCND1 & Neg. & Neg. & +++ & Neg. & + & Neg. & Neg. \\
\hline CD5 & + & +++ & +++ & Neg. & Neg. & Neg. & Neg. \\
\hline CD43 & + & +++ & +++ & Neg. & Neg. & Neg. & + \\
\hline CD23 & Neg. & +++ & Neg. & + & Neg. & Neg. & Neg. \\
\hline CD27 & ++ & +++ & +++ & +++ & Neg. & ++ & + \\
\hline Anexina A1 & Neg. & Neg. & Neg. & Neg. & +++ & Neg. & Neg. \\
\hline
\end{tabular}

CMF: citometria de fluxo; IHQ: imuno-histoquímica; LZME: linfoma da zona marginal esplênico; LLC/LL: leucemia linfoide crônico/linfoma linfocítico; LCM: linfoma de células do manto; LF: linfoma folicular; HCL: tricoleucemia; HCL-v: tricoleucemia variante; L.MALT: linfoma associado ao tecido linfoide da mucosa; Ig: imunoglobulina; Neg: negativo; +: raro; ++: algumas vezes; +++: sempre.

\section{Tabela 4 Marcadores indicados para a avaliação de linfomas/leucemias de células T/NK na MO}

\begin{tabular}{ll}
\hline Marcador & Expressão em células normais \\
CD3 & Células T, células NK \\
CD2 & Células T \\
CD5 & Células T \\
CD7 & Células T, células mieloides \\
CD8 & Células T citotóxicas \\
CD4 & Células T auxiliadoras, macrófagos \\
CD10 & Subgrupo de células B, subgrupo de células T \\
CD16 & Células NK \\
CD56 & Células T, células NK \\
CD57 & Subgrupo de células \\
Cadeia TCR-beta & Maioria das células T \\
CD25 & Células B e T ativadas \\
CD30 & Células B e T ativadas \\
TIA-1 & Grânulos citotóxicos \\
Granzima/perforina & Células citotóxicas T ativadas \\
Alk-1 & Não é expresso em células T normais \\
CD45R0 & Células T de memória, macrófagos \\
CD43 & Células T, células mieloides, subgrupo de células B \\
TdT & Células B e T precursoras \\
\hline
\end{tabular}

NK: natural killer; MO: medula óssea; TCR-beta: cadeia beta do receptor para antígeno da célula $T$. 
Tabela 5 Frequência do comprometimento da MO pelos processos linfoproliferativos de células T/NK

\begin{tabular}{|c|c|c|}
\hline $\begin{array}{l}\text { Apresentação } \\
\text { primária }\end{array}$ & $\begin{array}{c}\text { Tipo de } \\
\text { linfoma/leucemia }\end{array}$ & $\begin{array}{c}\text { Frequência de envolvimento } \\
\text { da MO (\%) }\end{array}$ \\
\hline Leucêmica/disseminada & $\begin{array}{l}\text { Leucemia prolinfocítica de células T } \\
\text { Leucemia de linfócitos T grandes granulares } \\
\text { Leucemia/linfoma de células T do adulto } \\
\text { Leucemia agressiva de células NK }\end{array}$ & $\begin{array}{l}>95 \\
>95 \\
60-70 \\
>95\end{array}$ \\
\hline Nodal & $\begin{array}{l}\text { Linfoma T angioimunoblástico } \\
\text { Linfoma de células grandes anaplásicas } \\
\text { Linfoma de células T periféricas, SOE }\end{array}$ & $\begin{array}{l}60-70 \\
15-25 \\
20-40\end{array}$ \\
\hline Extranodal & $\begin{array}{l}\text { Micose fungoide } \\
\text { LCGA primário da pele } \\
\text { Linfoma T subcutâneo paniculite-símile } \\
\text { Linfoma T tipo enteropático } \\
\text { Linfoma T hepatoesplênico } \\
\text { Linfoma T/NK extranodal tipo nasal }\end{array}$ & $\begin{array}{l}<5 \\
<5 \\
<5 \\
<5 \\
<95 \\
<5\end{array}$ \\
\hline
\end{tabular}

MO: medula óssea; NK: natural killer; SOE: sem outras especificações; LCGA: linfoma de grandes células anaplásicas.

vezes, infiltração sutil da $\mathrm{MO}$, que pode não ser detectada ao exame histológico convencional. A pesquisa de CD30, associada a EMA e ALK por meio de exame IHQ complementar, é essencial para o estadiamento correto desses pacientes, podendo aumentar a acurácia na detecção da infiltração linfomatosa, relacionada com pior prognóstico.

Uma observação importante refere-se à leucemia dos linfócitos T grandes granulares, que frequentemente passa despercebida ao exame morfológico da BMO, sendo caracterizada ao exame IHQ por uma população neoplásica intersticial e intrasinusoidal de células $T$ (CD3/CD8+) e por nódulos intertrabeculares de células $B$ circundados por células $T$ reativas (CD3/CD4+). Essa possibilidade deve ser considerada pelo patologista diante da biópsia de um paciente com granulocitopenia importante refratária ao tratamento.

Outro linfoma de células T que merece especial consideração é o angioimunoblástico, pois se apresenta, em geral, com acentuadas alterações inflamatórias e fibrose na região paratrabecular, obscurecendo as células T atípicas isoladas. Essas células $T$ apresentam imunofenótipo caracterizado por positividade para CD3/CD4/CD10/CXCL13. Outro fator de confusão diagnóstica é a presença de plasmócitos policlonais em número aumentado e células $B$ grandes CD20 positivas, que podem confundi-lo com o linfoma de grandes células $B$ rico em células T e histiócitos.

O linfoma de células $T$ hepatoesplênico é outra entidade que compromete a $\mathrm{MO}$ com padrão de infiltração sinusoidal, muitas vezes evidenciado apenas pela IHQ com marcadores de células T.

\section{Caracterização das doenças proliferativas mieloides}

Inúmeros marcadores que definem as linhagens celulares mieloides e suas diferentes etapas de maturação podem ser hoje identificados por IHQ ou CMF. Com isso, a imunofenotipagem das neoplasias derivadas dessas células inclui obrigatoriamente sua caracterização imunofenotípica.

\section{SMD/LAs}

Na SMD, a IHQ tem sido utilizada de rotina para duas finalidades:

- caracterização das três linhagens mieloides, auxiliando na identificação de sua desorganização arquitetural e formas atípicas/displásicas. São exemplos dessa situação a detecção de micromegacariócitos e a confirmação da linhagem granulocítica nos casos dos ALIPs, sigla para abnormal localization of immature precursors. Na identificação de células precursoras, caracterizam-se seu número e sua distribuição por meio dos marcadores CD34 e CD117. Deve-se lembrar que o CD117 também se expressa nos precursores eritroides. A tendência atual tem sido a de substituir a notificação dos ALIPs pela identificação de grupamentos multifocais de células progenitoras CD34+;

- diferenciação da forma hipocelular da SMD com a anemia aplástica. A imunomarcação com CD34 e Ki67 é muito útil para a identificação de células precursoras em atividade proliferativa, favorecendo 
significativamente o diagnóstico de SMD. Além disso, a identificação de micromegacariócitos também contribui para o diagnóstico de SMD.

O painel recomendado para a avaliação de um quadro suspeito ou definido de SMD deve incluir mieloperoxidase (MPO) (série granulocítica), glicoforina A (série eritrocítica), CD61 (ou CD42b, fator VIII; série megacariocítica), CD34 e CD117 (células precursoras/blastos).

A Tabela 6 mostra os marcadores mais utilizados para a linhagem megacariocítica e sua imunoexpressão em suas formas maturas e imaturas.

Nas LAs, um painel que inclua CD34, TdT, MPO, CD68 (KP-1) e CD68R (PGM-1), glicoforina A, CD61, CD42b, CD20, PAX5, CD79a, CD3 e CD1a é muito útil para a diferenciação das leucemias linfoides e mieloides, assim como para uma tentativa de subtipagem destas últimas segundo a classificação do French-American-British Group (FAB) (Tabela 7). As mutações recém-descritas no gene da nucleofosmina (NMP) na leucemia mieloide aguda (LMA) com cariótipo normal podem ser demonstradas por meio da expressão citoplasmática anormal da proteína pelo método IHQ com anticorpos anti-NMP. Da mesma forma, a coexpressão de marcadores B (CD79a e PAX5) caracteriza a LMA com a $\mathrm{t}(8 ; 21)$. Deve ser mencionado que, hoje, a CMF é considerada o método de eleição para a imunocaracterização das leucemias, sendo a IHQ utilizada para casos em que, por alguma razão, não é possível a obtenção de células viáveis para essa finalidade, como nas LMAs com fibrose (p. ex., leucemia megacarioblástica aguda), na panmielose aguda com mielofibrose e nas LMAs relacionadas com terapia.

\section{NMPC e NMP/MD}

O diagnóstico das NMPCs, como leucemia mieloide crônica (LMC), policitemia vera (PV), trobocitemia essencial (TE) e mielofibrose primária (MF), baseia-se geralmente em um conjunto de dados clínicos, hematimétricos, morfológicos e moleculares. A IHQ, incluindo glicoforina A ou C ou hemoglobina, MPO e CD61, pode, como na SMD, auxiliar na interpretação de células morfologicamente anormais, como os micromegacariócitos, e na estimativa de sua quantidade e sua distribuição. Além disso, CD34 pode ser útil para estimar o número de blastos, particularmente diante da suspeita de evolução para uma fase acelerada ou transformação aguda.

Como representante do grupo NMP/MD, a leucemia mielomonocítica crônica (LMMC) é caracterizada basicamente pelas informações obtidas com o exame do sangue
Anticorpos úteis para reconhecer

Tabela 6 megacariócitos atípicos maturos e formas blásticas

\begin{tabular}{l|c|c|}
\hline \multirow{2}{*}{ Anticorpo } & \multicolumn{2}{|c|}{ Megacariócitos } \\
\cline { 2 - 3 } & Maturos & \multicolumn{1}{|c|}{ Imaturos (blastos) } \\
\hline vWF & Bom & Inadequado \\
\hline CD42b & Bom & Bom \\
\hline CD61 & Bom & Bom \\
\hline CD31 (não & Bom & Bom \\
específico) & Pom & \\
\hline
\end{tabular}

Imunofenótipo em parafina: correlação

Tabela 7 limitada com os subtipos FAB da LMA

\begin{tabular}{|c|c|}
\hline$F A B$ & Marcadores \\
\hline MO & $\begin{array}{l}\text { CD34+; MPO+/-; CD68 (KP1)+/-; } \\
\text { CD68R (PGM-1)- }\end{array}$ \\
\hline $\mathrm{M} 1 / \mathrm{M} 2$ & $\begin{array}{l}\text { CD34+/-; MP0+; CD68 (KP1)+; CD68R } \\
\text { (PGM-1)- }\end{array}$ \\
\hline M3 & $\begin{array}{l}\text { MPO++; HLA-DR-; CD34-; CD68 } \\
\text { (PGM-1)- }\end{array}$ \\
\hline M4 & MP0+; CD68R (PGM-1)+/-; CD34- \\
\hline M5 & CD68R (PGM-1)+; MPO+/-; CD34+/- \\
\hline M6 & $\begin{array}{l}\text { Hb, glicoforina } A+>50 \% \text { das células; } \\
\text { MPO+/-; CD34+/- }\end{array}$ \\
\hline M7 & $\begin{array}{l}\text { CD61 > 50\% dos blastos; MPO-; } \\
\text { CD34+/- }\end{array}$ \\
\hline
\end{tabular}

FAB: French-American-British Group; LMA: leucemia mieloide aguda; MPO: mieloperoxidase; PGM-1: clone PGM-1; HLA-DR: human leucocyte antigen-DR; Hb: hemoglobina.

periférico e aspirado de MO. Quando a biópsia é realizada, o diagnóstico diferencial inclui LMC e LMC atípica (LMCa). A utilização do CD68 não tem sido de utilidade nessa distinção, utilizando-se CD68 (clone KP1), CD68R (clone PG-M1) ou CD163. Entretanto, a identificação dos blastos com CD34 pode auxiliar na classificação dos tipos 1 (<10\% de blastos) e 2 (10\%-19\% de blastos) ou na transformação franca para LMA (> 20\% de blastos). Além disso, CD123 tem sido útil para confirmar a presença dos nódulos de células dendríticas plasmocitoides, que só ocorrem na LMMC e não na LMC ou na LMCa. Esses nódulos mostram-se negativos para CD163 e irregularmente positivos para CD68 e CD68R.

Na mastocitose sistêmica, agora incluída no grupo das NMPCs na nova classificação da Organização Mundial da Saúde (OMS), 2008, a MO é um dos principais órgãos acometidos. Devido à fibrose, que se encontra geralmente 
presente nos focos de comprometimento pelos mastócitos, a biópsia tem sido o material mais eficiente para fins diagnósticos. Os mastócitos neoplásicos, assim como seus correspondentes normais, expressam CD117 e triptase de mastócitos. Entretanto, diferentemente das células normais, em geral, são hipo ou agranulados e expressam de forma aberrante CD25 e CD2.

\section{Investigação de doença metastática}

A MO é frequentemente sede de metástases de neoplasias de mama, pulmão e próstata nos adultos e neuroblastoma e rabdomiossarcoma em crianças. Os painéis de anticorpos devem ser definidos conforme a situação clínica, merecendo lembrar que algumas vezes a imunoexpressão de alguns anticorpos pode ser diferente no tumor primário e na doença metastática.

\section{Alterações reativas da MO - identificação de agentes infecciosos}

A IHQ pode auxiliar na identificação de agentes infecciosos, como o parvovírus humano B19, por meio da utilização de anticorpo específico (R92F6), além de outros agentes de infecções sistêmicas, como EBV, CMV, HHV8, micobactérias, protozoários e fungos.

\section{Conclusão}

O exame anatomopatológico da $\mathrm{MO}$ deve incluir, sempre que possível, o exame IHQ complementar, cujas indicação, seleção de anticorpos e interpretação exigem o conhecimento do contexto clínico, assim como informações de exames laboratoriais (incluindo sangue periférico), do esfregaço da $\mathrm{MO}$, citogenéticos e moleculares, além de imunofenotipagem por CMF.

\section{Referências}

1. DOGAN, A.; MORICE, W. G. Bone marrow histopathology in peripheral T-cell lymphoma. BJH, v. 127, p. 140-54, 2004.

2. DUNPHY, C. H. et al. Analysis of immunohistochemical markers in bone marrow sections to evaluate for myelodisplastic syndromes and acute myeloid leukemias. Appl Immunohistochem Mol Morphol, v. 15, p. 154-9, 2007.

3. FEND, F. et al. Modern techniques for the diagnostic evaluation of the trephine bone marrow biopsy: methodological aspects and application. Progress in Photochemistry and Cytochemistry, v. 42, p. 203-52, 2008.

4. FEND, F.; KREMER, M. Diagnosis and classification of malignant lymphoma and related entities in the bone marrow trephine biopsy. Pathobiology, v. 74, p. 133-43, 2007.

5. FRAGA, M. et al. Bone marrow involvement in anaplastic large cell lymphoma. Immunohistochemical detection of minimal disease and its prognostic significance. Am J Clin Pathol, v. 103, p. 82-9, 1995.

6. HORNY, H. P.; SOTLAR, K.; VALENT, P. Diagnostic value of histology and immunohistochemistry in myelodisplasic syndromes. Leukemia Research, v. 31, p. 1609-16, 2007.

7. KREMER, M. et al. Immunohistochemistry in bone marrow pathology: a usefull adjunt for morphologic diagnosis. Vrchows Arch, v. 447, p. 920-37, 2005.

8. MATUTES, E. et al. Splenic marginal zone lymphoma proposals for a revision of diagnosis and therapeutic criteria. Leukemia, v. 22, p. 487-95, 2008.
9. ORAZI, A. Histopathology in the diagnosis and classification of acute myeloid leukemia, myelodisplastic syndromes, and myelodisplastic/myeloproliferative diseases. Pathobiology, v. 74, p. 97-114, 2007.

10. ORAZI, A. et al. Chronic myelomonocytic leukemia: the role of bone marrow biopsy immunohistology. Mod Pathol, v. 19 , p. 1536-45, 2006.

11. OSUJI, N. et al. Characteristic appearances of the bone marrow in T-cell large granular lymphocyte leukemia. Histopathology, v. 50, p. 547-54, 2007.

12. TORLAKOVIC, E. E. et al. Call for a European programme in external quality assurance for bone marrow immunohistochemistry; report of a European Bone Marrow Working Group pilot study. J Clin Pathol, v. 62, p. 547-51, 2009. 